\title{
Long-term memory for pictures under conditions of thematically related foils
}

\author{
DONALD HOMA and CYNTHIA VIERA \\ Arizona State University, Tempe, Arizona
}

\begin{abstract}
Memory for pictures was investigated under conditions of difficult foil discriminability and lengthy retention intervals. The foils preserved the theme of the studied stimulus, but differed in the number and quality of nonessential physical details. In each experiment, subjects viewed colored photographs, black-and-white photographs, elaborated line drawings, and unelaborated line drawings, followed by an old/new (Experiment 1) or a four-alternative forced-choice (Experiment 2) test given either immediately, 1 day, 1 week, or 4 weeks following study; Experiment 3 replicated Experiment 1 but with a 12-week delay. For the old/new procedure, performance was best on colored photographs, with performance differences among the four stimulus types still significant after 4 weeks. For the forced-choice test, performance on colored photographs and unelaborated line drawings was best, with performance differences among stimulus types also still significant after 4 weeks. A confusion analysis indicated that errors were based on physical similarity, even after 12 weeks. These results refute the hypothesis that the memorial representations for pictorial variations converge to a common, thematic code after lengthy delays; instead, nonthematic, analogue information is encoded and preserved for lengthy time periods.
\end{abstract}

Results from a variety of studies have demonstrated that subjects are sensitive to both thematic and nonessential physical information in pictorial stimuli. When a subject was permitted to freely inspect a painting, Yarbus (1967) found that many of the subject's fixations occurred to five to six locations. With additional viewing time, many of the fixations were distributed not solely to novel areas but to the same five to six locations, often in the same order. Yarbus concluded that this scan path mirrored ongoing thought and was the subject's attempt to extract thematic information; fixations of novel areas reflected embellishments of the theme. Furthermore, the subject's scan path to the same picture could be markedly altered by changes in prior set, an outcome consistent with Yarbus's view that the scan path reflected theme extraction. In a somewhat different vein, Biederman and his coworkers (Biederman, 1972; Biederman, Glass, Rabinowitz, \& Stacy, 1974) found that the jumbling of a photograph differentially affected the subject's ability to identify thematic and object information. More recently, Biederman (1981) argued that thematic information is derived from both objects and the relations among those objects, where the latter may be characterized as involving either physical constraints (e.g., interposition) or referential meaning (e.g., probability). The deleterious effects of jumbling on thematic identification occur because object relations are violated.

The authors would like to thank Doug Grigoorio for his comments on an earlier version of this manuscript. Portions of this paper were presented at the 1987 Western Psychological Association Conference in Long Beach. The second author is presently supported through a fellowship from the Ford Foundation. Requests for reprints should be sent to Donald Homa, Department of Psychology, Arizona State University, Tempe, AZ 85287 .
What is interesting about memory for pictures is the potential role thematic and nonessential physical information might play in explaining the high levels of accuracy frequently found (e.g., Shepard, 1967; Standing, 1973). Advocates of dual-code theory (e.g., Bower, 1972; Paivio, 1971) explain picture memory by proposing that pictures are encoded in two distinct formats: a sensoryspatial-analogue code and a semantic-thematic-abstract code. The rich visual details of a picture are stored at a sensory level, and the meaning of a picture is stored semantically. Opponents of dual-code theory argue that subjects do not, in fact, remember the exact visual details and their spatial relations; instead, subjects remember a more abstract representation that captures the meaning of the picture (Anderson, 1985; Pylyshyn, 1984). In fact, Anderson (1978) demonstrated that dual-code and propositional theory can be framed so that their predictions are equivalent; that is, if neither theory is constrained, propositional explanations of picture memory cannot be distinguished from dual-code interpretations.

Regardless, theory development and refinement in this area have been hampered by the paucity of empirical data on the long-term retention of pictorial information, at least under conditions of difficult foil discriminability. In an experiment that closely mirrored some of the manipulations of the present study, T. O. Nelson, Metzler, and Reed (1974) had subjects first view black-and-white photographs, elaborated line drawings of these photographs, unelaborated line drawings of these photographs, or appropriate verbal descriptions of these photographs, followed by a two-alternative forced-choice test given immediately and 7 weeks after viewing. On the immediate test, recognition performance was equivalent for the four stimulus types; however, on the test given 7 weeks later, 
performance was significantly worse on the verbal descriptions and equal for the three picture versions. Had subjects stored the visual details available in the picture types, then performance might have been expected to covary with stimulus detail; that is, performance would have been best on the photographs, next on the elaborated line drawings, and worst on the unelaborated line drawings. Since performance on the three picture versions was equal, and since these versions differed in available visual detail but shared a common theme, support for a nonsensory, propositional explanation seemed clear.

However, there are at least three reasons why this interpretation should be questioned: (1) each subject worked with only one of the stimulus types, (2) the foils were semantically unrelated to the studied photographs, and (3) the immediate test results were not replicated in a subsequent study that employed the same stimuli (Loftus \& Bell, 1975). For the purposes of the present study, the first two criticisms are especially important. First, because the degree of discriminability required to distinguish old and new stimuli may not have been equivalent for the different stimulus types, potential performance differences among the three picture formats may have been masked by differences in foil discriminability. In addition, by using only unrelated foils, it is impossible to determine whether performance was mediated by correct retrieval of thematic or extraneous physical information. In particular, T. O. Nelson et al. (1974) could not test a strong prediction of a semantic-only hypothesis: if subjects store only the meaning of a stimulus, can they later discriminate that stimulus from a foil that is theme-preserving but differing in detail? The failure to replicate the immediate test results by Loftus and Bell might be due to a number of factors, including the fact that performance was at ceiling (95\%-100\%) in the Nelson et al. study. Unfortunately, Loftus and Bell did not include a delayed test.

Results from recent studies have failed to clarify how pictures are represented in memory. Intraub and Nicklos (1985) found that memory for detailed colored photographs was better if attention was drawn to physical details rather than to meaning details, an outcome opposite that obtained with verbal materials. Similarly, the picture superiority effect (greater memory for pictures than verbal equivalents) is reduced when a conceptually dissimilar study set is drawn to be schematically similar (D. L. Nelson, Reed, \& McEvoy, 1977; D. L. Nelson, Reed, \& Walling, 1976). These studies also employed an immediate test only.

What is needed is an assessment of picture memory over lengthy time periods, where the foils are thematically similar but differ in detail. Under such conditions, three general predictions can be assessed. If only meaningpreserving information is stored, then performance should be equivalent on picture types that differ only in nonessential physical detail, both immediately after as well as after lengthy delays. (Performance should also be quite poor, since originals and theme-preserving foils should be nearly impossible to discriminate.) If both thematic and extraneous visual information are stored, then perfor- mance should be monotonic with the amount and quality of detail contained in the picture. ${ }^{1}$ Finally, if both thematic and nonessential physical detail are initially encoded but the extraneous visual detail is rapidly forgotten (or forgotten at a faster rate than thematic information), then the initial performance advantage for stimuli high in detail should vanish with sufficiently long retention intervals.

These predictions were assessed in the present study using four types of pictorial stimuli: colored photographs, black-and-white versions of the colored photographs, elaborated line drawings of the colored photographs, and unelaborated line drawings of the colored photographs. At the time of study, the subject saw different pictures in each of these formats. At the time of test, the subject had to discriminate between studied items and foils that were thematically similar, where the foils differed from the original in terms of number and quality of nonessential physical details. (Experiment 1 included three rating tasks that evaluated the importance of various details to the pictorial theme and the similarity among the four versions.) The foils differed from their studied versions in terms of color (e.g., a colored photograph was transformed to its black-and-white equivalent), shading, and background objects and fine-grained detail. To the extent that thematic detail was held constant between the study item and test foil, correct discrimination would reflect preservation of extraneous physical detail. These predictions were evaluated both immediately following viewing and at delays as long as 4 (Experiments 1 and 2) and 12 (Experiment 3) weeks.

One other manipulation was used. Recognition performance on the stimulus variations might differ, depending upon how recognition was tested. In the present study, the processes or subject strategies evoked by a yes/no and by a forced-choice test might be different, since the memory demands may not be the same. On a fouralternative forced-choice test, the studied item is included among three foils that differ only in detail. Selection of the correct alternative may occur because this version evokes a greater similarity or familiarity match with the memorial representation than any of the alternatives. Importantly, only the versions contained in the array need be considered. ${ }^{2}$ In contrast, an old/new test places a greater (and different) burden on memory. To reject a foil in an old/new task, the subject must retrieve information sufficient to reject the stimulus. This could involve either retrieving details not included in the presented foil (e.g., retrieving color information to reject a black-and-white foil) or by deleting details from the presented foil (e.g., rejecting a colored foil after studying the black-and-white version). Importantly, the subject must generate and evaluate details retrieved from memory rather than evaluating perceptually available alternatives (as is the case in a forced-choice test). Bartlett, Gernsbacher, and Till (1987) argued that picture memory involves a mixture of familiarity and image sampling processes. In our experiment, old/new testing may be more likely to induce evaluation based on image sampling, whereas forced-choice testing may induce decisions based primarily on familiar- 
ity. Regardless, both kinds of tests were used, following an identical study session: in Experiments 1 and 3, an old/new procedure was used, whereas a four-alternative forced-choice test was used in Experiment 2. Potential differences in strategy for these two types of tests are discussed in more detail in the General Discussion.

\section{EXPERIMENT 1}

\section{Method}

Subjects. A total of 81 introductory psychology students served as subjects to complete the course requirements. Twenty subjects were randomly assigned to one of four different delay groups: immediate, 1 day, 1 week, and 4 weeks. One subject in the immediate delay group was replaced because her scores were 3.94 standard deviations below the group mean. The subjects were tested in groups of 5 .
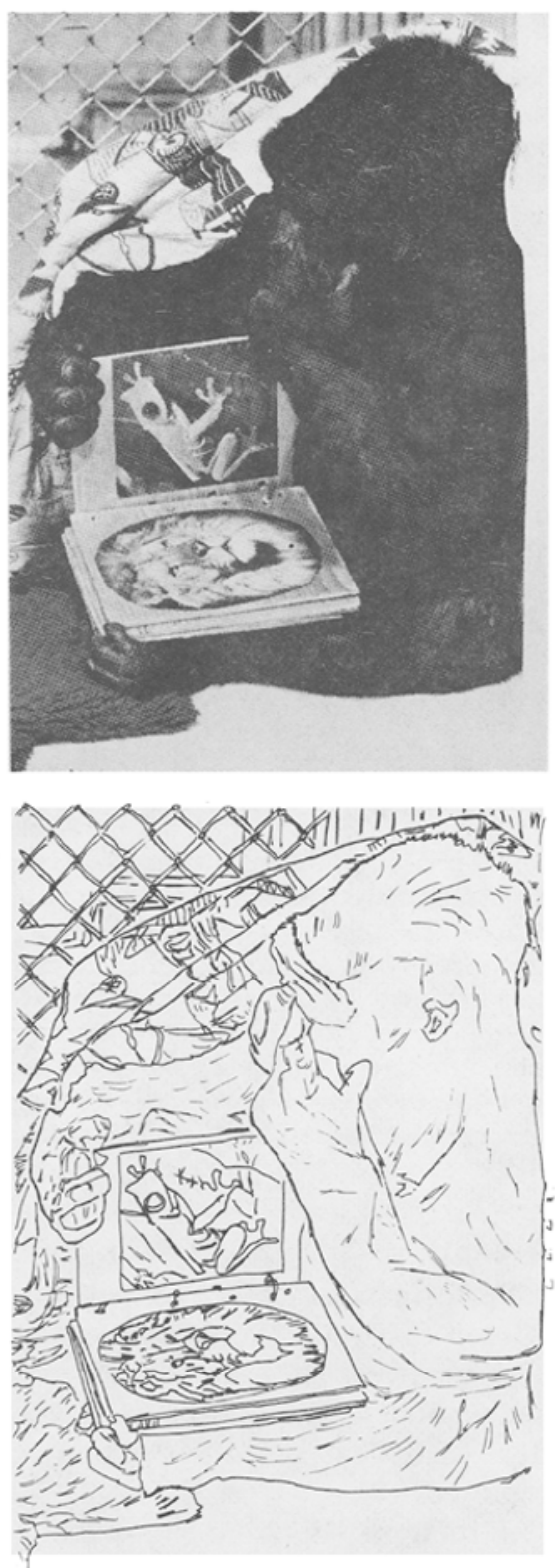

Figure 1. An example of the four stimulus versions. Note: The picture in the upper left quadrant was printed in color.
Stimuli preparation. The final set of stimuli consisted of 96 colored photographs, each depicting a salient theme and nonessential detail, and three sets of variations. For each colored photograph (C), three versions were prepared: a black-and-white copy (BW), an elaborated line drawing (E), and an unelaborated line drawing (U). The elaborated line drawing was generated by tracing over the colored photograph and included the nonessential detail but not the shading or fine-grained detail. The unelaborated line drawing was generated by tracing over the elaborated line drawing so as to preserve the salient theme of the original colored photograph but eliminating all nonessential detail. The final set of stimuli (four sets of 96 items) were made into $35-\mathrm{mm}$ slides. An example of one picture's four versions is shown in Figure 1.

Stimuli validation. Two separate validation procedures were performed to verify that our manipulation of detail did not modify or alter the theme of each scene across the four stimulus versions. A third rating procedure was performed to assess the perceived similarity among the four stimulus versions. In the first procedure,
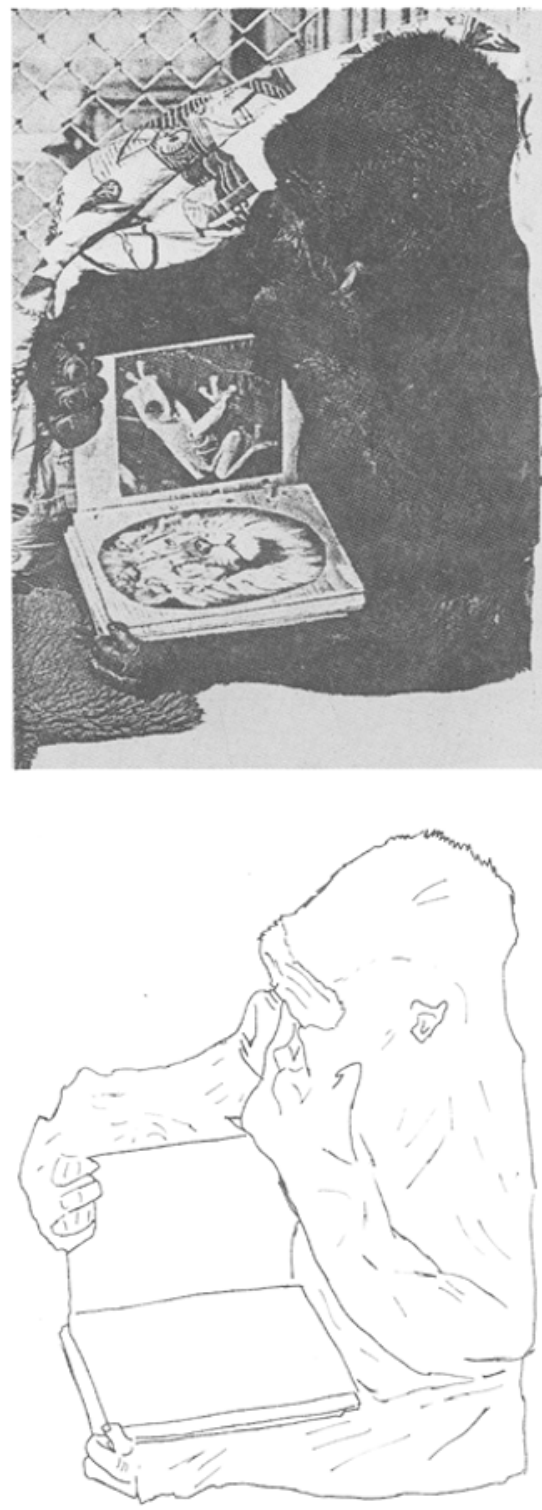
10 subjects were shown a randomly selected subset of 24 of the colored slides and were asked to rate the importance of various components of the picture to the theme. Four components were selected for rating based on the detail eliminated from the colored version to derive each of the three variations. Subjects used an 11-point scale, in which $0=$ This detail has no importance to the theme and $10=$ This detail is extremely essential to the theme. The subjects were asked to rate the importance of the vivid colors (absent in the black-and-white version), of the dark and light tones and the shading (missing in the elaborated version), of the entire background (additionally absent in the unelaborated version), and of the central figure(s) judged by the authors to be the central focus or theme of each scene (and the only items contained in the unelaborated version). To ensure that raters understood what details were being rated, the experimenter pointed to each of the four components specifically for each of the 24 slides, one at a time, in random order across the entire subset, giving subjects time to inspect the picture and rate each component separately before proceeding to the next component. The results of this rating task supported our attempt to construct theme-preserving variations: the mean ratings for importance of the theme, color, shading, and background components were $8.98,5.41,6.30$, and 6.11, respectively. An item analysis revealed that the theme rating was significantly greater than the other three $(p<.001)$ and that the latter three ratings did not differ from each other $(p>.20){ }^{3}$

In a second validation procedure, subjects rated how well a validated verbal description of an unelaborated line drawing matched the central focus or theme of each of the picture versions. First, the verbal description validation procedure that was originally used in a different study is described. Different groups of 10 subjects described the meaning or theme of each of the four versions of each of the 96 scenes. These descriptions were then used to derive a composite description for all 384 slides. Different groups of 10 subjects were then shown one of the four versions of each of the 96 scenes and were asked to select which of the four descriptions matched the presented slide. Thus, each distinct group of subjects in this part of the procedure saw only one of the four pictorial versions and were not informed that three other pictorial versions existed. A description was deemed acceptable only when 6 out of 10 subjects correctly matched the description to the appropriate slide. Any descriptions failing to reach this criterion were reviewed and modified and resubmitted to the same procedure using a completely new group of subjects. Using a binomial distribution, correct matches for 6 of 10 subjects with chance selection at .25 is significantly better than chance at $p<.05$. A randomly selected subset of the unelaborated verbal descriptions for $\mathbf{4 8}$ different scenes, validated by the above procedure, was then used in the present rating study as descriptions of the central theme of each scene.

A completely new group of 13 subjects was then presented with each of the 48 theme descriptions, paired with one of the four versions of that scene, and was asked to rate how well the slide depicted the information contained in the description. Twelve of the 48 picture-description pairs were distractors in which the theme described a totally different scene. Twelve slides appeared in each of the four stimulus types. A 5-point scale was used in which a rating of 1 indicated that the meaning of the description did not match the meaning in the picture and a rating of 5 indicated that the description and the picture matched very well. These ratings confirmed that we had effectively preserved the theme of each scene in all four visual versions: mean ratings were $1.25,4.32,4.44$, 4.16, and 3.98 for the mismatched, colored, black-and-white, elaborated, and unelaborated versions, respectively. An item analysis indicated that the only significantly different ratings were produced by the mismatched pairs $(p<.001)$. Mean ratings on the four stimulus versions did not differ from each other $(p>.10)$.

Finally, similarity ratings were obtained from an additional group of 12 subjects who were not involved in any other aspect of this experiment. A different randomly selected sample of 48 scenes was used, with 8 different scenes assigned to each of the six pair combinations (C-BW, C-E, C-U, BW-E, BW-U, E-U). Each pair of slides was presented simultaneously using two Kodak Carousel projectors, and subjects were asked to rate how similar the slides were to each other using a 5-point scale in which $1=$ very dissimilar and $5=$ very similar. Mean ratings for the six pairs, C-BW, BW-E, C-E, E-U, BW-U, and C-U, were 4.70, 4.28, 4.17, 3.65, 3.46 , and 3.44, respectively. An item analysis (using a significance criterion of $p<.05$ ) revealed that the items in the C-BW combination were rated as significantly more similar to each other than were those in the E-U, BW-U, and C-U combinations. Furthermore, the C-E pairs were rated as significantly more similar to each other than were the BW-U and C-U pairs; and the BW-E pairs received significantly higher similarity ratings than did the $\mathrm{C}-\mathrm{U}$ and BW-U pairs. All other comparisons received statistically equivalent ratings $(p>.05)$.

Stimuli assignment. Items were counterbalanced across subjects using a $4 \times 4$ Latin square, so that each item was presented for study in each of the four formats in all four delays. Four different presentation orders were randomly generated so that 5 subjects at each delay received the same presentation order during study. Additionally, four different presentation orders were randomly generated and used in a similar manner for the test presentations.

Design and Procedure. A 4 (delay) $\times 4$ (stimulus type) mixed design was used, with the delay factor as a between-subjects variable and stimulus type as a within-subjects variable.

During study, all subjects were shown all 96 themes, 24 in each of the four formats, for $5 \mathrm{sec}$ each. The subjects were seated in a semicircle approximately $6 \mathrm{ft}$ from a white projection screen suspended from the wall. Those subjects in the immediate condition were given an intervening arithmetic task for approximately 12-15 min immediately following the study session while the test slides were being arranged. The testing procedure for the four delays was otherwise identical.

At test, the subjects were again shown all 96 items, $50 \%$ in the same format as during study, and the remainder as foils, equally distributed among the three remaining formats. Thus, of the 24 items originally viewed as colored slides, 12 appeared again as colored slides during the test session. Of the remaining 12, 4 appeared as black-and-white slides, 4 as elaborated line drawings, and 4 as unelaborated line drawings. This procedure was replicated for all four formats.

The subjects were informed prior to the test that $50 \%$ of the stimuli would appear in the same format as during study and that the remaining 50\% were equally likely to appear in any of the other three formats. The subjects were told to answer "old" only to those items that appeared in exactly the same format as during study, and to respond "new" otherwise (identifying as old any nonstudied version was scored as a false alarm). Furthermore, subjects were required to give a confidence rating for each answer using a scale of $1-3$, where $1=$ uncertain, $2=$ moderately certain, and $3=$ very certain. The test items were presented at the rate of $5 \mathrm{sec}$ per slide.

\section{Results}

Performance measures based on hit rates, a $d^{\prime}$ analysis (Green \& Swets, 1966), and an accuracy measure combining correct recognitions and correct rejections (with and without the confidence judgments) all resulted in identical statistical outcomes. Analyses based on $d^{\prime}$ are reported.

$d^{\prime}$ analysis. Figure 2 shows the mean $d^{\prime}$ score for each stimulus type as a function of the four delays. In general, performance decreased across the 4-week delay by 


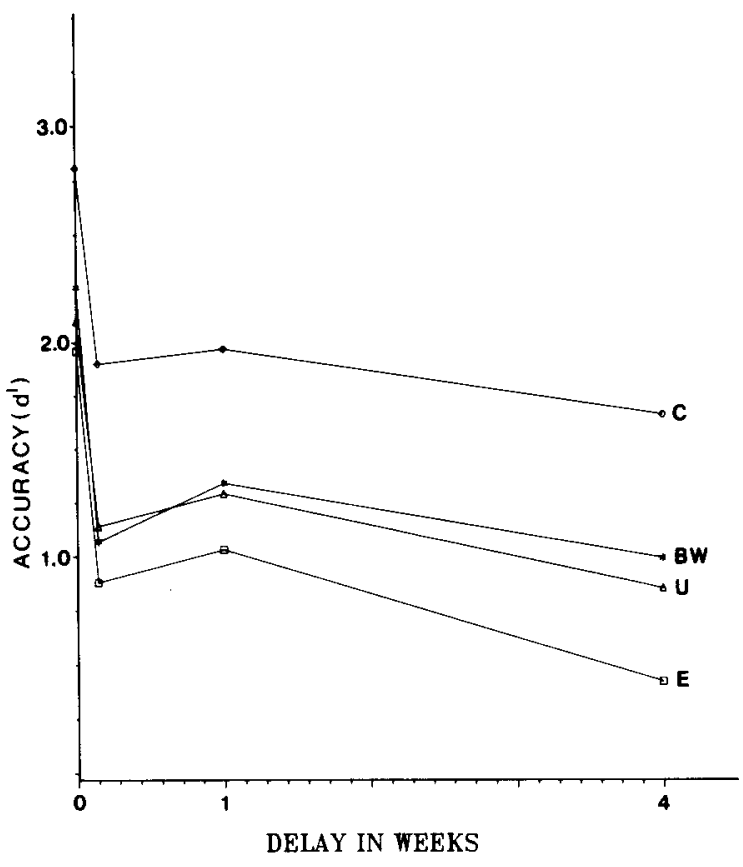

Figure 2. Mean $d^{\prime}$ for each stimulus type as a function of retention interval in Experiment $1 . \mathrm{C}=$ colored photograph; $\mathrm{BW}=$ black-and-white photograph; $\mathbf{E}=$ elaborated line drawing; $U$ = unelaborated line drawing.

$50 \%-70 \%$, with overall $d^{\prime}$ scores highest for the colored photograph (2.08) relative to the other stimulus types (black-and-white $=1.42$; unelaborated $=1.34$; elaborated $=1.07$ ). The main effects of delay and stimulus type were both significant $[F(3,76)=24.00$ and $F(3,228)$ $=32.46$, respectively; each $p<.001]$. The interaction between delay and stimulus type was not significant $(p>.10){ }^{4}$ Post hoc Tukey tests revealed that performance on the colored format exceeded performance on the remaining stimulus types $(p<.01)$, with performance on black-and-white and unelaborated better than that on elaborated $(p<.05)$. A simple analysis on only the immediate test showed that the superiority of the colored format was evident on this test $(p<.001)$.

The decrease in performance across delay was characterized by comparable changes in both hits and false alarms; overall, hits decreased by .150 (immediate $=$ $.778 ; 4$ weeks $=.628$ ), and false alarms increased by .153 (immediate $=.157 ; 4$ weeks $=.310$ ). Even after 4 weeks, performance on each stimulus type was better than chance ( $p<.01$ in each case).

Confusion analysis. A confusion analysis was done to determine whether the tendency for false alarms was a function of the study-test format, and whether these rates changed over time. For example, 24 colored photographs were presented in the study phase, and 12 appeared as old items in the test phase. The other 12 studied photographs appeared equally often as foils in the other three formats. Table 1 shows the false alarm rates for each study and test format at each delay, conditionalized upon an error; the chance confusion rate is .33 .
We were initially interested in whether the two pictorial versions ( $C$ and $\mathrm{BW}$ ) and the two line drawings ( $\mathrm{E}$ and $\mathrm{U})$ would be confused with each other at rates greater than chance. (In the General Discussion, we consider two alternative definitions of similarity, one based on transformation distance and one based on pairwise similarity ratings.) Of the 16 contrasts of interest ( $C$ with $\mathrm{BW}, \mathrm{BW}$ with $C, E$ with $U$, and $U$ with $E$ at each of the four delays), 11 were significant at $p<.01$ (z-approximation to a binomial); only 1 contrast not involving these pairs was significant ( $\mathrm{E}$ for $\mathrm{BW}$ after 4 weeks). When the confusion rates for these 4 contrasts were collapsed at each delay, each delay produced statistically significant outcomes ( $p<.01$ in each case). For example, when the black-and-white photographs were studied and an immediate test was provided, $52.5 \%$ of all false alarms occurred to the colored versions $(z=2.58, p<.01)$. Similarly, elaborated and unelaborated line drawings were frequently confused, regardless of delay.

These results support the conclusion that confusions were not random but were related to the similarity of the study format. Interestingly, when colored photographs and unelaborated line drawings were studied, the best foil type remained the same across the four delays (for colored photographs, the best foils throughout were the black-andwhite photographs; for the unelaborated line drawings, the best foils throughout were the elaborated line drawings). In contrast, the best foil for black-and-white photographs and elaborated line drawings changed over time.

Table 1

False Recognition Confusions, Conditional Upon an Error, Experiment 1

\begin{tabular}{|c|c|c|c|c|}
\hline \multirow{2}{*}{$\begin{array}{c}\text { Study } \\
\text { Stimulus }\end{array}$} & \multicolumn{4}{|c|}{ Test Stimulus } \\
\hline & $\mathrm{C}$ & BW & E & $\mathbf{U}$ \\
\hline \multicolumn{5}{|c|}{ Immediate } \\
\hline $\mathrm{C}$ & - & $.434 *$ & .247 & .319 \\
\hline BW & $.525^{*}$ & - & .250 & .225 \\
\hline E & .156 & .221 & - & $.624 *$ \\
\hline $\mathrm{U}$ & .118 & .176 & $.706^{*}$ & - \\
\hline \multicolumn{5}{|c|}{ 1-Day Delay } \\
\hline $\mathrm{C}$ & - & $.492 *$ & .297 & .261 \\
\hline BW & .361 & - & .347 & .292 \\
\hline $\mathrm{E}$ & .250 & .280 & - & $.470^{*}$ \\
\hline $\mathbf{U}$ & .246 & .261 & $.493^{*}$ & - \\
\hline \multicolumn{5}{|c|}{ 1-Week Delay } \\
\hline $\mathrm{C}$ & - & $.471^{*}$ & .279 & .250 \\
\hline BW & .354 & - & .369 & .277 \\
\hline E & .217 & .317 & - & $.467 *$ \\
\hline $\mathrm{U}$ & .235 & .235 & $.529^{*}$ & - \\
\hline \multicolumn{5}{|c|}{ 4-Week Delay } \\
\hline $\mathrm{C}$ & - & $.462 *$ & .231 & .308 \\
\hline BW & .284 & - & $.500^{*}$ & .216 \\
\hline $\mathrm{E}$ & .254 & .373 & - & .373 \\
\hline $\mathrm{U}$ & .183 & .338 & $.479 *$ & - \\
\hline
\end{tabular}

Note-C = colored photograph; BW = black-and-white photograph; $\mathrm{E}=$ elaborated line drawing; $\mathrm{U}=$ unelaborated line drawing. ${ }^{*} p<.01$. 
For example, the best foil on an immediate test for the black-and-white photographs was a colored photograph, but after 1 month, the best foil was an elaborated line drawing. Finally, confusion rates deviated from chance levels for each stimulus type even after 1 month, except for the elaborated stimulus version.

\section{Discussion}

Recognition accuracy was significantly better for colored photographs, relative to the other formats, regardless of time of test. Furthermore, performance differences among the four stimulus types were significant and of similar magnitude throughout the retention interval, which suggests that the codes representing each stimulus type were discriminable and stable; that is, initial representations did not converge into a common format, at least not after a delay of 4 weeks. Further support for the stability of each format across delay was found in the confusion analyses: even after 4 weeks, confusion rates were significantly related to foil similarity.

In Experiment 2, a four-alternative forced-choice test replaced the old/new task used in Experiment 1. This task should maximize discrimination difficulty, since the correct choice was always paired with its three variations. To the extent that irrelevant detail is rapidly forgotten over time, then performance should rapidly decline into a common level at near-chance levels. Should performance on each stimulus type again be above chance level at each delay, and should performance differences among the stimulus types be manifested after 4 weeks, then evidence for a stable representation based on distinguishing visual detail would again be found.

\section{EXPERIMENT 2}

\section{Method}

Subjects. A different group of 92 introductory psychology students served as subjects in Experiment 2. A total of 12 subjects was replaced (an entire group of 5 was replaced due to the interruption of the testing session by one subject who was ill and wished to discontinue the session; an additional 7 subjects were replaced for failure to follow instructions). Subject assignment was identical to Experiment 1.

Stimuli. The same four stimulus sets from Experiment 1 were used in the same manner during the study session. A fifth set of slides consisting of 95 of the original 96 themes was prepared in the form of a four-item array containing the four different stimuli versions. (The absence of one theme from the test set was due to the inadvertent failure to photograph the test array for that theme.) For all 95 test slides, the array format was as follows: the colored version was shown in the upper left quadrant, the black-and-white version in the upper right quadrant, the elaborated line drawing in the lower left quadrant, and the unelaborated line drawing in the lower right quadrant.

Design and Procedure. The design and study procedure were identical to Experiment 1. At test, the subjects were shown all 95 test arrays and were instructed to identify the stimulus they had viewed during the study session. They were informed that during the study session, approximately $25 \%$ of the stimuli had appeared in each of the four formats, and that, for each test array, a colored photograph would appear in the upper left quadrant, a black-andwhite photograph in the upper right quadrant, an elaborated line drawing in the lower left quadrant, and an unelaborated line drawing in the lower right quadrant.

The subjects were instructed to carefully inspect each test array and to indicate which of the four stimuli had been viewed at study by recording their choice on prepared scoresheets. They were then told to indicate their confidence, for their first choice only, by using the 1-3 confidence scale described in Experiment 1. After deciding their first choice and assessing their confidence, the subjects were then told to rank-order the remaining formats from most likely to least likely. If the subjects were unable to rank-order their remaining choices, they could ignore them. However, they were instructed that for each trial they must always indicate their first choice and their confidence rating for that choice. ${ }^{5}$

The subjects were told that they would have about $10 \mathrm{sec}$ on each trial and that, prior to the end of the trial, they would hear the word "respond." If they had not made a response by that time, they were then to write down their choice, guessing if necessary. Care was taken to ensure that all subjects understood the instructions prior to commencing the test session. The test items were presented at a rate of about 11-12 sec per item.

\section{Results}

Performance measures based on hit rates, a $d^{\prime}$ analysis, and an accuracy measure combining correct recognition and correct rejections all resulted in identical statistical outcomes. Analyses based on $d^{\prime}$ are reported.

$d^{\prime}$ analysis. Figure 3 shows the mean $d^{\prime}$ score for each stimulus type as a function of the four delays. In general, performance decreased across the 4-week delay by about $60 \%$, with overall $d^{\prime}$ scores highest for the colored photographs (1.56) and unelaborated drawings (1.56), relative to those for the other two stimulus types (black-andwhite $=1.04$; elaborated $=1.32$ ). The main effects of delay and stimulus type were both significant $[F(3,76)=$

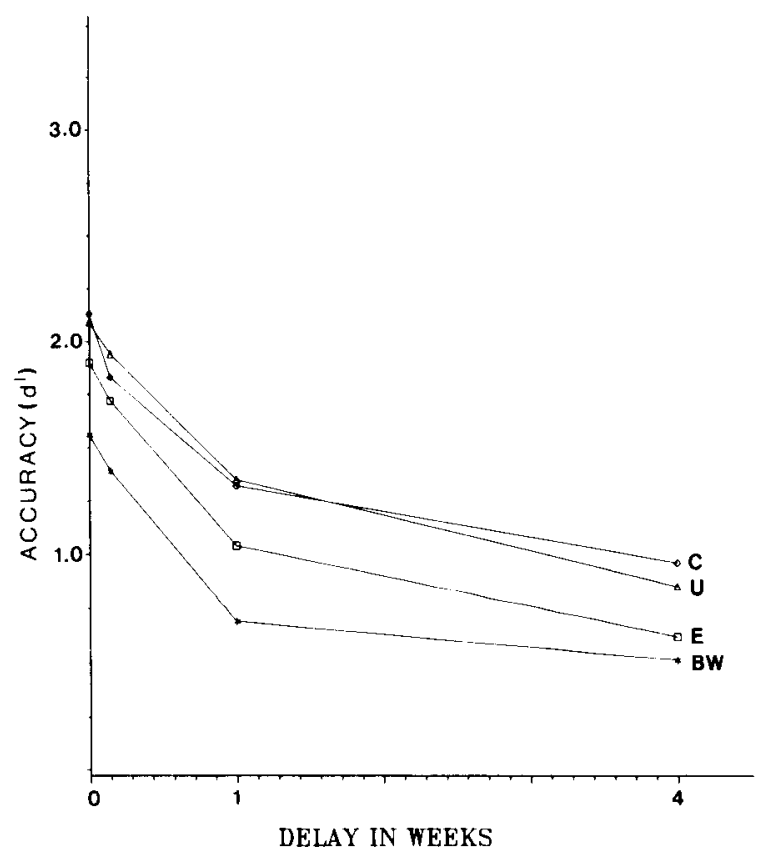

Figure 3. Mean $d^{\prime}$ for each stimulus type as a function of retention interval in Experiment 2. $\mathrm{C}=$ colored photograph; $\mathrm{BW}=$ black-and-white photograph; $\mathbf{E}=$ elaborated line drawing; $\mathrm{U}=$ unelaborated line drawing. 
21.35 and $F(3,228)=35.91$, respectively; each $p<.001]$. The interaction between delay and stimulus type was not significant $(p>.10)$. Post hoc tests revealed that performance on the colored format and unelaborated format did not differ $(p>.10)$, but both exceeded performance on the remaining stimulus types $(p<.01)$, with elaborated better than black-and-white $(p<.01)$.

The hit rate decreased by about $30 \%$ for each stimulus type across the 4-week delay (immediate $=.716 ; 4$ weeks $=.430$ ). Nonetheless, performance on each stimulus type was better than chance even after 4 weeks $(p<.01)$. For example, poorest performance was obtained on the blackand-white photographs after 4 weeks, yet all 20 subjects provided a positive $d^{\prime}$ value for this stimulus type after 4 weeks.

Confusion analysis. Table 2 shows the confusion rate for each study and test format, as a function of delay (conditionalized upon an error). As was the case in Experiment 1 , confusion rates appeared to be systematically related to the original study format, even after 4 weeks. For the same 16 contrasts of interest (C with $\mathrm{BW}, \mathrm{BW}$ with $C, E$ with $U$, and $U$ with $E$ at each delay), 14 were significant at $p<.01$; none of the other contrasts was significant. The confusion rate for the best foil did show some decline across the retention interval (immediate = $.504 ; 1$ day $=.539 ; 1$ week $=.460 ; 4$ weeks $=.432$ ), but these rates are still above chance, even for the 4-week delay $(z=5.93, p<.01)$.

Table 2

False Recognition Confusions, Conditional Upon an Error, Experiment 2

\begin{tabular}{|c|c|c|c|c|}
\hline \multirow{2}{*}{$\begin{array}{c}\text { Study } \\
\text { Stimulus }\end{array}$} & \multicolumn{4}{|c|}{ Test Stimulus } \\
\hline & C & BW & $\mathbf{E}$ & $\mathbf{U}$ \\
\hline \multicolumn{5}{|c|}{ Immediate } \\
\hline C & - & $.487^{*}$ & .313 & .201 \\
\hline BW & $.500 *$ & - & .300 & .200 \\
\hline $\mathbf{E}$ & .103 & .369 & - & $.528 *$ \\
\hline $\mathbf{U}$ & .152 & .345 & $.502 *$ & - \\
\hline \multicolumn{5}{|c|}{ 1-Day Delay } \\
\hline C & - & $.530 *$ & .232 & .238 \\
\hline BW & $.520^{*}$ & - & .259 & .239 \\
\hline $\mathbf{E}$ & .193 & .284 & - & $.572^{*}$ \\
\hline $\mathbf{U}$ & .104 & .361 & $.535^{*}$ & - \\
\hline \multicolumn{5}{|c|}{ 1-Week Delay } \\
\hline $\mathrm{C}$ & - & $.556 *$ & .204 & .240 \\
\hline BW & .372 & - & .372 & .376 \\
\hline $\mathbf{E}$ & .205 & .336 & - & $.459^{*}$ \\
\hline $\mathbf{U}$ & .154 & .392 & $.454^{*}$ & - \\
\hline \multicolumn{5}{|c|}{ 4-Week Delay } \\
\hline C & - & $.430^{*}$ & .321 & .249 \\
\hline BW & $.430^{*}$ & - & .300 & .270 \\
\hline $\mathbf{E}$ & .212 & .342 & - & $.445 *$ \\
\hline $\mathbf{U}$ & .225 & .389 & .386 & - \\
\hline
\end{tabular}

Note-C = colored photograph; BW = black-and-white photograph; $\mathrm{E}=$ elaborated line drawing; $\mathrm{U}=$ unelaborated line drawing. ${ }^{*} p<.01$.

\section{Discussion}

The results of Experiment 2 were similar to those obtained in Experiment 1, with one major exception: performance was best for the colored photographs and unelaborated drawings (in Experiment 1, performance on the unelaborated line drawings was poor). Otherwise, no evidence for convergence among the four types across the delay was obtained, which again argues against a convergence to a common code for the stimulus types. This conclusion is supported by the confusion data, which again show that study-test confusions were significantly related to similarity even after 4 weeks.

\section{EXPERIMENT 3}

Experiment 3 attempted to replicate the results of Experiment 1 (especially the ordering of stimulus types), and investigated the potential for convergence among stimulus codes for a far greater delay (12 weeks rather than 4 weeks). In addition, a within-subjects manipulation of delay was used, with different sets of stimuli used on the immediate and delayed test.

\section{Method}

Subjects. A total of $\mathbf{4 0}$ introductory psychology students served as subjects, none of whom had served in the previous two experiments.

Stimuli. The same stimulus assignment used in Experiment 1 was used in Experiment 3, with half of the stimulus set (48 items) used in the immediate test and the remaining 48 used in the 12-week delay condition. The four stimulus types were counterbalanced across subjects over the two delays, using a $4 \times 4$ Latin square design similar to that in Experiment 1. Eight different presentation orders were randomly generated and used during the study session, and an additional set of eight different presentation orders were randomly generated and used during the test session.

Design and Procedure. A 2 (delay) $\times 4$ (stimulus type) withinsubjects design was used. Study and test procedures were identical to those in Experiment 1, with the exception that no confidence ratings were collected in Experiment 3.

\section{Results}

Performance measures based on hit rates, $d^{\prime}$, and recognition accuracy all resulted in identical statistical outcomes. Analyses based on $d^{\prime}$ are reported.

$d^{\prime}$ analysis. Figure 4 shows the mean $d^{\prime}$ score for each stimulus type as a function of delay. In general, performance decreased by about $80 \%$ at the 12-week delay, with overall $d^{\prime}$ scores highest on the colored photographs (2.02), relative to the other stimulus types (black-andwhite $=1.33$; elaborated $=1.01$; unelaborated $=1.12$ ) . The main effects of delay and stimulus type were both significant $[F(1,78)=146.57$ and $F(3,156)=16.98$, respectively; each $p<.001]$. The interaction between delay and stimulus type was not significant $(p>.10)$. Post hoc tests revealed that performance on the colored format exceeded performance on the remaining stimulus types $(p<.01)$, with performance on the remaining formats not differing from each other $(p>.10)$. 


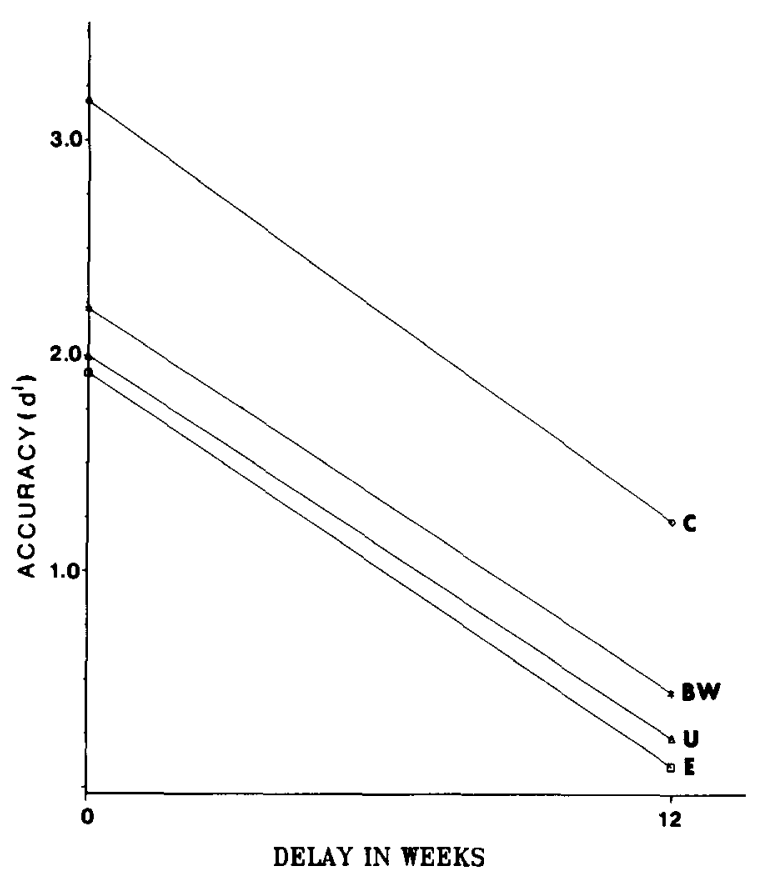

Figure 4. Mean d' for each stimulus type as a function of retention interval in Experiment 3. $\mathrm{C}=$ colored photograph; $\mathrm{BW}=$ block-and-white photograph; $E$ = elaborated line drawing; $U=$ unelaborated line drawing.

Hit rates decreased by about $30 \%$ across the 12 -week delay (immediate $=.780 ; 12$ weeks $=.492$ ), and false alarms increased by about $16 \%$ over the same period (immediate $=.184 ; 12$ weeks $=.348$ ). Although the performance drop was considerable across 12 weeks, performance was still above chance, with 34 of the 40 subjects having positive $d^{\prime}$ values $(p<.01)$. Of the four stimulus types, only the elaborated drawings functioned at a chance level after 12 weeks $\left(d^{\prime}=0.10, p>.10\right)$.

Confusion analysis. Table 3 shows the rates of false alarms for each stimulus type as a function of the format originally studied, shown separately for immediate and delayed tests. As was the case in the previous experiments, false alarms on the immediate test were strongly related

Table 3

False Recognition Confusions, Conditional Upon an Error, Experiment 3

\begin{tabular}{lcccc}
\hline \multirow{2}{*}{$\begin{array}{c}\text { Study } \\
\text { Stimulus }\end{array}$} & \multicolumn{4}{c}{ Test Stimulus } \\
\cline { 2 - 5 } C & \multicolumn{5}{c}{ BW } & E & U \\
\hline C & - & $.742^{*}$ & .194 & .065 \\
BW & .367 & - & $.429^{*}$ & .204 \\
E & .229 & .208 & - & $.562^{*}$ \\
U & .255 & .191 & $.553^{*}$ & - \\
C & & $12-$ Week Delay & & \\
BW & - & .302 & .245 & $.453^{*}$ \\
E & .196 & - & .280 & .258 \\
U & $.449 *$ & .370 & - & $.435^{*}$ \\
\hline
\end{tabular}

Note-C = colored photograph; BW = black-and-white photograph; $\mathbf{E}=$ elaborated line drawing; $\mathbf{U}=$ unelaborated line drawing. ${ }^{*} p<.01$. to the visual similarity between original and test formats (collapsed across the four critical contrasts, $z=5.72$, $p<.01$ ); on the test 12 weeks later, false alarm rates were a bit erratic but, overall, were still significantly related to visual similarity $(z=2.46, p<.01)$. The major difference on the delayed test was the reduced false alarm rate to black-and-white photographs when the studied item was a colored photograph, and the reduced rate to unelaborated drawings when the original was an elaborated drawing. As was the case in Experiment 1, there was a high false alarm rate to colored photographs when black-and-white photographs were studied, and unelaborated drawings were frequently called old when elaborated stimuli were studied. Overall, it would appear that the false alarm rate to visually similar items is reduced after 12 weeks, relative to that on an immediate test, but that the rate is still greater than chance.

\section{Discussion}

The results of Experiment 3 largely replicated those found in Experiment 1. Therefore, the performance ordering in an old/new test appears to be a reliable one, and one that is unchanged across delays as long as 12 weeks after study. Furthermore, no evidence for convergence to a common code was apparent after 12 weeks, and confusions based on visual similarity were apparent, albeit reduced, even 12 weeks after study.

\section{GENERAL DISCUSSION}

Three major results emerged from the present study: (1) subjects encode sufficient pictorial detail in a single 5 -sec viewing period to reject thematically related foils over time periods as long as 12 weeks later, (2) the memorial representation of thematically related stimuli does not converge to a common code after lengthy delays, and (3) the type of test strongly influences performance on stimulus types.

In each experiment, performance on the four stimulus types was above chance, with the sole exception of the elaborated line drawings in Experiment 3 at the 12-week delay. These results are all the more remarkable, given that subjects observed each of 96 stimuli for only $5 \mathrm{sec}$ and were later required to discriminate the studied stimuli from theme-preserving foils after substantial delays. At a minimum, subjects clearly store more than core or thematic information; some degree of extraneous physical detail is also encoded and retained. The existence of significant performance differences among the stimulus types after 12 weeks suggests that information does not decay into a common, thematic code, at least not within this time period. Further support for the durability of distinctive codes comes from the kinds of false alarms made by subjects. Even after 12 weeks, errors occur disproportionately to visually similar foils. There is a suggestion that the rate of visually based confusions slowly declines with time; only large-scale parametric tests (varying study time and using longer delays such as 6 months) would verify whether this trend is specious or real. Regardless, 
the stability of confusions and the long-term performance differences for pictures varying in extraneous physical detail seem at variance with studies using verbal materials. For example, the convergence of performance on verbatim and gist recall after brief delays (less than $1 \mathrm{~h}$ ) has been obtained frequently in studies of sentence memory (e.g., Reder, 1982). These studies typically use short sentences with little detail, in which subjects are asked to discriminate tense change and/or changes in which foils contain the subjects or verbs from other studied sentences. We are currently investigating memory for short passages that have substantial embellishment of detail. Under these conditions, retention for detail might better mimic that found for pictures.

It is incorrect to conclude that these results favor a dualcode interpretation to a propositional one, since, without theoretical constraints, visually based representations can be mapped onto propositionally based ones, and vice versa (Anderson, 1978). Nonetheless, these results place severe constraints on the kind of theory (propositionally based or visually based) needed to explain these findings. A propositional theory that states that only core information is either initially stored or available after a short duration (e.g., 1 h) is clearly incorrect. Anderson (1985) contended that, "subjects are not likely to remember the exact visual details or spatial relations in a picture. Instead, they are remembering some rather abstract representation that captures the picture's meaning..." (p. 107). The results of the present study suggest that this contention is misleading and probably incorrect. Rather, the propositional code must be sufficiently complex to accommodate both thematic and extraneous visual detail for time periods as long as 4-12 weeks after brief inspection.

The results obtained with the two paradigms (old/new and forced-choice) did not produce the same ordering for the various stimulus types. This outcome, although unexpected, appears to be stable, at least insofar as the color version is concerned, since the ordering obtained in Experiment 1 was similar to that in Experiment 3. In Experiments 1 and 3, an old/new procedure resulted in an ordering that was generally consistent with the quantity of details available at study: colored photographs were recognized more accurately than black-and-white photographs, which enjoyed a slight (nonsignificant) advantage over the line drawings (performance on elaborated and unelaborated was equivalent in Experiment 3, but unelaborated produced a significant advantage in Experiment 1). In Experiment 2, a four-alternative forced-choice test favored colored photographs and unelaborated line drawings, followed by elaborated drawings and black-andwhite photographs. Since the experiments were otherwise identical, an explanation based on differences in strategy or processing seems likely.

In an old/new task (Experiments 1 and 3), the subject must evaluate the match between the current stimulus and its memorial representation. Except for cases in which the subject failed to encode the study item and/or failed to retrieve its representation at test, the match between a test probe and its representation had to always be posi- tive, since all foils were variations on studied stimuli. To evaluate the goodness of the current match, the subject must generate, from memory, variations of the current stimulus probe by adding or deleting details. If any of the generated matches exceeds the similarity of the current probe with its internal representation, then the subject is likely to respond that the stimulus is new. Importantly, the generated matches are likely to exceed the match of the current stimulus only if the subject adds/deletes the correct detail. For example, the subject presented with an elaborated line drawing at the time of test might first match this stimulus with its memorial trace. If the original (study) stimulus was a colored photograph of the same theme, successful rejection of the current probe requires that the subject retrieve the appropriate coloration of the stimulus. The successful addition of detail is required whenever the test probe is less complex than its studied variation. When the test probe is more complex than its studied variation, correct rejection requires the proper deletion of detail. Thus, a test probe that appears as a colored photograph can be rejected as new only if the subject correctly deletes the proper detail (e.g., removing color if the original probe was a black-and-white photograph). A number of subjects wrote comments reflecting the addition/deletion of details, for example, "I remember that the dress was deep blue" (addition of detail). Since accuracy in the old/new procedure favored performance on the more detailed stimuli, and since this advantage was uniformly maintained across lengthy delays, it must be concluded that the additional details inherent in the more complex stimuli were encoded and faithfully preserved over time.

In contrast, the forced-choice test (Experiment 2) makes generation of alternatives from memory unnecessary since the choices for evaluation are presented in the test array. On a forced-choice test, the subject may compute similarity matches for each test variation, identifying as old the one version receiving the highest score. What needs explanation is the ordering of performance of the variations, especially in light of the different ordering on the old/new task. One possibility is that interpicture similarity (Experiment 1) determined both the ordering and the rate of confusion among the four versions. In fact, the rated similarity was high for C-BW and BW-E pairs. This would explain why subjects frequently incorrectly selected the black-and-white version after studying the color version, and why performance on the black-and-white stimuli was poor (two similar foils exist). However, rated interpicture similarity cannot explain why subjects, after studying the elaborated version, more often selected the unelaborated version than the colored or black-and-white versions ( $E-U$ was rated as less similar than either $E-C$ or E-BW), or why the elaborated version was the primary false alarm when the unelaborated version was studied (the unelaborated version was equally similar to the other three versions).

An alternative possibility is that the colored photographs and unelaborated line drawings, which occupy extreme positions on an assumed scale of complexity, were sub- 
ject to less interference/competition than the two intermediate variations. For example, colored photographs differ from black-and-white photographs only in the absence of color; from elaborated line drawings in terms of color and precision of detail; and from unelaborated line drawings in terms of color, precision of detail, and number of details. This is consistent with the ordering of the mean similarity ratings obtained on the pairwise comparisons involving the color stimulus version. In effect, the colored photographs contained features that differed from the foils in one, two, or three ways or transformations (color, precision, quantity). A similar argument may be made for the unelaborated line drawings: its foils also vary in one, two, or three ways. However, both the blackand-white photographs and the elaborated line drawings have two foils that differ in only one way. For example, an elaborated line drawing differs from a black-and-white photograph in terms of precision of detail, and from an unelaborated line drawing in terms of quantity of detail. The existence of two highly similar foils for the elaborated line drawings and the black-and-white photographs, versus the existence of one highly similar foil for the colored photographs and the unelaborated line drawings, may be the basis for the superior performance on colored photographs and unelaborated drawings. Note that this rationale, which predicts the obtained ordering on the forced-choice test (Experiment 2), is inappropriate as an explanation for the old/new task, since, in the latter, the addition/deletion of details must be generated from memory. If the full complement of alternatives is not generated, and/or the accuracy of retrieved details is imperfect, then the number of close foils in the old/new task may be functionally smaller than in the forced-choice task.

Although neither paradigm produced evidence of convergence to a common, thematic code after 4-12 weeks, one could argue that the distinction between thematic and extraneous physical detail is not as great as supposed (in spite of evidence to the contrary noted in the rating tasks described in Experiment 1). This argument might suggest that the full complement of visual details are not incidental attributes, but part of the intrinsic core meaning of a stimulus. Thus, color may produce moods that modify the core meaning or theme of a picture, as might the very angularity/curvilinearity/texture of details. Differences among picture types after lengthy delays were obtained, according to this argument, not because nonessential detail was encoded and preserved, but because subtle thematic differences existed among the stimulus variations, which permitted later discrimination. This argument would assert that each picture has a unique thematic interpretation, and that variations of this picture are not variations in detail but variations in theme. Acceptance of this argument (that thematic and physical distinctions are illusory), however, carries a theoretical cost: memory representations for pictorial stimuli (propositional or otherwise) must be remarkably complex and sensitive to visual detail, since variations of visual nuances can be discriminated even 12 weeks later. The results of this study support either of these interpretations: either thematic and extraneous physical details are encoded and stored in durable form (without convergence to a common thematic code over time), or what is stored and preserved is a richly detailed thematic interpretation that is sensitive to shading, texture, color, and so on. Either interpretation is contrary to the assertion that only the core meaning is extracted and preserved from the visual array, and that incidental details are rapidly forgotten.

\section{REFERENCES}

ANDERSON, J. R. (1978). Arguments concerning representations for mental imagery. Psychological Review, 85, 249-277.

ANDERSON, J. R. (1985). Cognitive psychology and its implications. New York: Freeman.

Bartlett, J. C., Gernsbacher, M. A., * Till, R. E. (1987). Remembering left-right orientation of pictures. Joumal of Experimental Psychology: Leaming, Memory, \& Cognition, 13, 27-35.

Biederman, I. (1972). Perceiving real-world scenes. Science, 177, 77-80.

Biederman, I. (1981). On the semantics of a glance at a scene. In K. Kubovy \& J. R. Pomerantz (Eds.), Perceptual organization. Hillsdale, NJ: Erlbaum.

Biederman, I., Glass, A. L., Rabinowitz, J. C., Stacy, E. W., JR. (1974). Searching for objects in real-world scenes. Journal of Experimental Psychology, 97, 22-27.

BowER, G. H. (1972). Mental imagery and associative learning. In L. Gregg (Ed.), Cognition in learning and memory. New York: Wiley.

GrEen, D. M., \&WETS, J. A. (1966). Signal detection theory and psychophysics. New York: Wiley.

INTRAUB, H., \& NiCKLOS, S. (1985). Levels of processing and picture memory: The physical superiority effect. Journal of Experimental Psychology: Learning, Memory, \& Cognition, 11, 284-298.

LofTUs, G. R. (1985). Evaluating forgetting curves. Journal of Experimental Psychology: Learning, Memory, \& Cognition, 11, $397-406$.

LoFTUs, G. R., BELL, S. M. (1975). Two types of information in picture memory. Joumal of Experimental Psychology: Human Learming \& Memory, 104, 103-113

Nelson, D. L., Reed, V. L., \& McEvoy, C. L. (1977). Learning to order pictures and words: A model of sensory and semantic encoding. Journal of Experimental Psychology: Human Leaming \& Memory, 3, 485-497.

Nelson, D. L., Reed, V. L., \&alling, J. R. (1976). Pictorial superiority effect. Joumal of Experimental Psychology: Human Learning \& Memory, 2, 523-528.

Nelson, T. O., Metzler, J., \& Reed, D. A. (1974). Role of details in the long-term recognition of pictures and verbal descriptions. Journal of Experimental Psychology, 102, 194-196.

Paivio, A. (1971). Imagery and verbal processes. New York: Holt, Rinehart \& Winston.

Pylyshyn, Z. W. (1984). Computation and cognition. Cambridge, MA: MIT Press.

Reder, L. M. (1982). Plausibility judgment versus fact retrieval: Alternative strategies for sentence verification. Psychological Review, $89,250-280$.

SHEPARD, R. N. (1967). Recognition memory for words, sentences, and pictures. Journal of Verbal Learning \& Verbal Behavior, 6, 155-163.

StANDING, L. (1973). Learning 10,000 pictures. Quarterly Journal of Experimental Psychology, 25, 207-222.

YARBUS, A. L. (1967). Eye movements and vision. (B. Haigh, Trans.). New York: Holt, Rinehart \& Winston.

\section{NOTES}

1. This assumes that the subject would encode more details for the more detailed stimuli, that encoding was not constrained by variables such as exposure time and contrast, and that the additional encoded details were used in subsequent judgments such as foil rejection. 
2. This is an implicit assumption of most forced-choice tests and one that was made explicit in the present study. In fact, one could construct a forced-choice test that included some, but not all, alternatives, and/or presented foils that differed from the original in both type (e.g., color vs. black and white) and distortion of type (e.g., a colored foil of an originally studied colored stimulus). Manipulation of the latter type of foil would provide a fine-grained test of the amount and quality of preserved detail.

3. We cannot conclude, on the basis of these ratings, that the color, shading, and background details were irrelevant to the theme, since these components received values greater than 0 . However, we also do not know what rating corresponds to psychological zero on this scale (subjects tend to avoid the endpoints of a scale). The safest conclusion is that the intended theme was generally rated as very thematic by the subjects, and that the other components were rated equally and considerably less important to the theme. This issue is addressed in the General Discussion.
4. One of the reviewers (Geoffrey Lofus) correctly noted that the interpretation of statistical interactions as a basis for inferring differential forgetting rates is fraught with problems, especially when performance measures (e.g., $\left.d^{\prime}\right)$ are nonlinearly related to each other (e.g., the hit rate) (Loftus, 1985). This problem is further complicated when performance differences exist at the shortest delay. For our purposes, the stability of different codes finds support in the significant differences among stimulus types at the longest delay.

5. Having subjects rank-order the remaining alternatives after they made their first selection resulted in many blank or undecipherable responses for the rank-order data. For that reason, that data are not reported here. The confidence data for the first selection did not suffer from these scoring problems, but the analysis of confidence data produced statistical outcomes identical to those reported in the Results section.

(Manuscript received March 5, 1987; revision accepted for publication January 12,1988 .) 